\title{
Transdisciplina, Enseñanza en Posgrados, Escuela Superior De Administración Pública (ESAP)
}

Escobar, Ricardo Antonio; Escobar, María Beatriz

Transdisciplina, Enseñanza en Posgrados, Escuela Superior De Administración Pública (ESAP)

Revista Educación, vol. 44, núm. 1, 2020

Universidad de Costa Rica, Costa Rica

Disponible en: http://www.redalyc.org/articulo.oa?id=44060092032

DOI: https://doi.org/10.15517/revedu.v44i1.37336

Esta obra está bajo una Licencia Creative Commons Atribución-NoComercial-SinDerivar 3.0 Internacional. 


\section{Transdisciplina, Enseñanza en Posgrados, Escuela Superior De Administración Pública (ESAP)}

\section{Transdisciplinarity and Postgraduate Teaching, Higher School of Public Management, (ESAP)}

Ricardo Antonio Escobar

scuela Superior de Administración Pública, Colombia

ricardo.escobar@ucm.edu.co

iD http://orcid.org/0000-0003-2865-9715

Maria Beatriz Escobar

Universidad Católica de Manizales, Caldas, Colombia

maescobar@ucm.edu.co

iD http://orcid.org/0000-0002-0419-9032
DOI: https://doi.org/10.15517/revedu.v44i1.37336

Redalyc: http://www.redalyc.org/articulo.oa?id=44060092032

Recepción: 24 Junio 2019

Aprobación: 03 Diciembre 2019

\section{Resumen:}

La enseñanza del saber administrativo está caracterizada por poca investigación, interés en la construcción disciplinar y restringida proyección social. Se pretende mejorar las prácticas de enseñanza de la administración, integrando la pedagogía como elemento para humanizar. Esta investigación presenta una metodología cualitativa, transdisciplinar de investigación acción que tiene etapa diagnóstica en donde se indaga con una encuesta por el pre saber del cuerpo docente, personal administrativo y estudiantes; en la segunda etapa, una sesión en profundidad crea un espacio de interlocución, y en la tercera, un taller de aprendizaje transdisciplinar, resultando en espacios donde los tres estamentos se integran y construyen las propuestas pedagógicas; en conclusión la transdisciplina es un concepto que enriquece la aproximación pedagógica y el saber administrativo. El conocimiento transdisciplinar contribuye en que la escuela de administración se asuma como un escenario de acogida.

Palabras Clave: Aprendizaje, Docencia, Administración, Disciplina escolar, Proceso de comunicación, Transdisciplinariedad, Pensamiento complejo.

\section{ABstract:}

Management Education is characterized by scarce research, interest in disciplinary development and little social projection. This is a qualitative, cross-disciplinary research that consisted of a diagnostic phase during which faculty, management staff and students were asked to complete a survey. The second phase involved an in-depth group discussion and the third phase a cross-disciplinary learning workshop. The results of the three sectors of the survey were used to develop pedagogical recommendations to enrich pedagogy and Management Studies at the Management School.

KEYWORDS: Learning, Teaching, Management, School Discipline, Communication Process, Transdisciplinarity, Complex Thinking.

\section{INTRODUCCIÓN}

La enseñanza de la administración se mueve en un devenir histórico en el que los espacios y la temporalidad cambian en función de la construcción de los contextos económicos, sociales y culturales donde se encuentran inmersos sus saberes, sus técnicas y sus prácticas; esta movilidad tiene como lugar epistémico común un juego contradictorio de apuestas ideológicas, posturas metodológicos y constructos conceptuales sobre los cuales se elaboran y reelabora las perspectivas que se asumen del acto pedagógico y de la didáctica propia de la disciplina. Enseñar es un hecho que une trasversalmente las interacciones y las relaciones que se dan como 
parte de una dinámica en la que participan docentes, personas administrativas y estudiantes de la escuela de administración.

Las prácticas de enseñanza se asumen en un espacio que determina claramente las concepciones teóricas y científicas en las que históricamente se pueden enmarcar la evolución y la trasformación del pensamiento administrativo (Taylor, Fayol, y Lender, 2003; Ponce, 1985; Weber,1994), establecieron las bases de una administración científica, centrada en la división del trabajo, la jerarquización del mando, el control del tiempo, la optimización de los recursos, la distribución de las tareas según áreas y departamentos, en el marco de una estructuración burocrática de las empresas.

Las teorías clásicas comienzan a transformarse con la emergencia de otros enfoques y tendencias, que pretenden explicitar la presencia del ser humano y de los factores motivacionales en el logro de rendimiento, productividad y rentabilidad de las organizaciones. Los sistemas de administración fundamentados en las relaciones humanas, encuentran en las contribuciones de Mayo (1946) y de McGregor (1969) Un claro alejamiento de las concepciones reductivas que ponen de relieve las tareas los tiempos y los movimientos. Esta perspectiva asigna enorme valor a factores psicológicos como la motivación y la comunicación en la construcción de dinámicas de grupo interpersonales que fortalecen las capacidades humanas y potencia una variedad de habilidades que incrementan la productividad como resultado de los refuerzos positivos y la estimulación permanente de los grupos humanos.

Una tercera línea de los enfoques administrativos que suelen enseñarse se encuentra asociada a la apropiación del concepto de management, cuyo hilo conductor está dado por la propuesta de una administración por objetivos, que optimiza el planeamiento estratégico y construye sinergias colectivas a partir de un liderazgo transformacional. Los teóricos como: Ponce (1985), Musgrave y Elster (1974), Odiorne (1995) y Steiner (1985) incorporan en la administración estilos de liderazgo que incrementan la productividad creando una dinámica organizacional coherente con las necesidades de la empresa, a partir de una gerencia exigente y la definición de áreas claves que pueden robustecerse para lograr una mejora continua. Los objetivos y las metas son básicos para crear visión y misiones compartidas que se constituyen en el punto de partida para una implementación estratégica que sea al mismo tiempo competitiva, humana y altamente motivadora.

Las prácticas de enseñanza construidas sobre la base de estos enfoques generan la visión fragmentada del conocimiento (Chaves, 2010). De la administración, acentúa los dualismos, la binariedad y ausentan al ser humano como sujeto de la ciencia y activo participante en la comprensión integradora y compleja de sus procesos y objetos de estudio. Los modelos administrativos terminan por convertirse en construcciones estandarizadoras y que se rigen por una pretensión universalista que instrumentaliza la racionalidad administrativa y deshumaniza del saber que circula en la proyección social de sus conocimientos (Vélez, 2011).

Como precedente de la investigación desde el pensamiento complejo, se tiene la desarrollada por Espinosa (2013) donde a través de una investigación-acción con actores (docentes, estudiantes y directivos/as) de las distintas áreas del CEUArkos de Puerto Vallarta, Jalisco, México, se construyó y llevó a la práctica estrategias metodológicas para operacionalizar la transdisciplinariedad y la complejidad en el aprendizaje, la enseñanza y la investigación.

Situando la investigación en el contexto específico de la escuela de administración superior ESAP de la ciudad de Manizales, Álvarez (2010) considera que la enseñanza del saber administrativo está caracterizada por un pobre desempeño, la ausencia de investigación, poco interés en la construcción disciplinar y una restringida proyección social. Estas circunstancias inconvenientes generan la necesidad de fundamentar una perspectiva transdisciplinaria del saber administrativo, cimentada en una aproximación compleja a la realidad que es multidimensionada y policromática en sus distintos niveles de percepción; el logro de esta construcción transdisciplinar remite a un interés gnoseológico por elaborar una propuesta pedagógica que integre el diálogo de saberes, la apertura metodológica y la pedagogía como un terreno en construcción de 
prácticas humanizadoras que le aporten al cumplimiento de los objetivos misionales de una universidad que le apuesta a la calidad y a la excelencia.

\section{Marco teórico}

$\mathrm{Al}$ iniciar la investigación en el año 2015 se efectúo una pesquisa teórica, reflexiva y profunda de lo que significa e implica la investigación transdisciplinaria como opción metodológica, pero en las bases de datos el concepto de transdisciplina aparece muy poco y más si se combina con la palabra administración, solo unas cuantas publicaciones se refieren al tema, por lo cual se podría decir que para describir la administración con su transdisciplinariedad se debe observar desde los Centros de Educación Superior donde se lleva a cabo la formación de estudiantes.

El abordaje de esta investigación busca determinar aquellas aproximaciones teóricas y metodológicas, las cuales de algún modo introducen lenguajes emergentes en la enseñanza de la administración, la formación de los profesionales, el papel de la educación superior y la apropiación social de la producción científica, se publicó el artículo titulado, el pensamiento transdisciplinar en la enseñanza de la administración una pesquisa por su estado del arte entre el año 2000 y 2015 en donde se ejecutó una búsqueda de los artículos, Escobar y Escobar, (2016) encontraron:

En las bases de datos Scielo, Pubmed, Lilacs, Biblioteca Virtual de Salud, ProQuest, Ebrary, Elibro, Medline, Google Academic, Redalyc, Hinary, Scopus y ScienceDirect. Se obtuvieron en total 78 artículos de los cuales 49 fueron seleccionados, se agruparon tres cortes cronológicos a saber: 2000-2005, 2006-2010, 2011-2015.Finalmente, se concluye que la emergencia de nuevos lenguajes conduce a la aceptación de la transcomplejidad, como una nueva episteme para abordar los procesos que son inherentes a la reflexión por la importancia del ser y el quehacer científico, pedagógico y social de la administración (p. 1).

Se promueve el pensamiento transdisciplinar desde un proceso participativo con actores universitarios, esta es una población que presenta diversidad de disciplinas como economía, administración pública, psicología, e incluso otras que están por fuera de la educación y la pedagogía como la ingeniería química, las artes plásticas y la geología. Esta variedad de especificidades de teóricas y prácticas, genera la necesidad de efectuar un abordaje transdisciplinario de la realidad en la cual se encuentran inmersos docentes, estudiantes y personal administrativo. Esta descripción es el punto de partida para comprender que la transdisciplina es fundamental de la comprensión de las relaciones que se dan entre el conocimiento, el ser humano y los diferentes contextos donde este se produce. Desde esta perspectiva, la transdisciplina al asumirse como metodología, se encuentra fundamentada en lo que Nicolescu (2006) denomina tres pilares, los cuales son enunciados por él como los niveles de Realidad, la lógica del tercero incluido y la complejidad, estos determinan la metodología de la investigación transdisciplinaria.

Estos tres pilares, se presume, deben visibilizarse en el desarrollo de la propuesta didáctica como parte de la investigación que se plantea. Los niveles de realidad, se constituyen en las instancias que le permiten a cada actor universitario construir miradas multiperspectivas del ejercicio de su disciplina, la didáctica, la enseñanza y el aprendizaje. Estas visiones comportan nuevas lógicas que según Nicolescu (2009) los espacios entre las diferentes disciplinas, en los cuales la mirada unidimensional es sustituida por abordajes mucho más amplios, en relación a lo cual puntualiza, en contraste con la realidad de un solo nivel y unidimensional del pensamiento clásico, la transdisciplinariedad reconoce la multidimensionalidad de la realidad (Carrizo, 2005). Al plantear una propuesta didáctica para promover pensamiento transdisciplinar entre diferentes actores universitarios que se mueven en los espacios de postgrado en una disciplina como la alta gerencia de la economía pública, lo que se intenta es que estos se motiven para efectuar un abordaje teórico y práctico de su disciplina desde una visión transdisciplinaria que considera importante porque la visión transdisciplinaria elimina la homogeneización, y reemplaza la reducción con un nuevo principio de realidad que emerge de la coexistencia de una pluralidad compleja y una unidad abierta (Carrizo, 2005). 
Se presenta la ciencia moderna tradicional antepuesta a las ideas de la complejidad y transdisciplina como maneras de abordar y generar conocimiento desde la perspectiva de los sistemas complejos. Se proponen opciones metodológicas, epistémicas y teóricas (Benítez y López, 2017).

La importancia de las disciplinas, al asumirlas desde la perspectiva transdisciplinaria, cobra una mayor preponderancia, por cuanto, se les mira en contextos y situaciones de pluralidad, complejidad y apertura. Los diálogos se hacen necesarios para que cada profesional en educación pueda cambiar conceptos y prácticas, las que, al ser incorporadas en sus procesos didácticos, de enseñanza y aprendizaje, podrían mejorar significativamente. El diálogo aparece como indispensable para involucrar a cada participante en una acción reflexiva que transforme su modo de comprender dicha realidad el sujeto se introduce progresivamente en un proceso dialógico y reflexivo, que implica la expansión, y reorganización de la conciencia (Pozzoli, 2006).

La reflexión y la acción son procesos segmentarios que proponen otras dimensiones de la realidad, de las cuales es preciso que los actores universitarios intenten hacerse conscientes. Solo así se hace posible determinar algunos problemas que están incidiendo en la enseñanza y en el aprendizaje, en relación con el desarrollo didáctico de cada disciplina. Emerge, la necesidad de incorporar la lógica del tercero incluido porque de este principio depende la promoción de un pensamiento transdisciplinar, mediante el cual los actores universitarios puedan establecer la coherencia entre los distintos niveles de la realidad de la coherencia y la interacción (Nicolescu, 2006).

Este es un desafío de enorme significado, el cual es enunciado por Morin (1998) en las siguientes palabras el desafío de la complejidad es el de pensar complejamente como metodología de acción cotidiana, cualquiera sea el campo en el que se desempeña el quehacer profesional. No es una tarea fácil lograr sensibilizar un grupo heterogéneo de personas para pensar complejamente, porque cada uno tendrá o asume diferentes miradas de su mundo personal, del conocimiento en el que se ha especializado, de la enseñanza y de los procesos de aprendizaje.

Se trata de incorporar la lógica de la complejidad, como un aspecto importante para comprender las relaciones que se dan entre todos los niveles de realidad que están implicados en la enseñanza y el aprendizaje de la alta gerencia en economía pública. Por ejemplo, aprender a dimensionar adecuadamente la coexistencia de conceptos como organización, capital intelectual, desarrollo local y global, solo por citar algunos con los que actores universitarios se enfrentan durante el desarrollo académico del posgrado.

La complejidad es básica para que se pueda establecer la coherencia entre estos conceptos con la realidad misma de la cual forman parte, ya sea cuestionándola o problematizándola para alcanzar miradas multiperspectiva (Nicolescu, 2009).

El principio sistémico desempeña un papel importante en la construcción de la propuesta, porque mediante su utilización se le da forma a una nueva organización de los saberes teórico-prácticos. Esta tiene como punto de partida el diálogo entre las disciplinas y la mirada compleja de la realidad en sus diferentes interdependencias coherencia e interacciones. Esto no significa que se desconozcan conceptos como diversidad, heterogeneidad o individualidad; por el contrario, la teoría de los sistemas complejos planteada por García (2006), contribuye a enriquecer los diálogos y las comprensiones.

El pensamiento transdisciplinar, presume la creación de puentes de comunicación entre las disciplinas, en un proceso de construcción permanente, involucrando las subjetividades humanas (Codina, Sotolongo, y Díaz, 2006) desde una perspectiva hologramática, lo hologramático es definido como lo que se "convierte en relevante el constante diálogo entre lo macro y lo micro" (Morin, 2004, p. 25). Cuando se intenta poner a dialogar disciplinas tan diferentes como la economía, la administración o la sicología, esta perspectiva hologramática es de gran importancia para que el conocimiento no se diluya en posibles fragmentaciones o reducciones. Esta mirada se hace mucho más pertinente, en el momento que se intenta sistematizar una propuesta didáctica para que los procesos de enseñanza y aprendizaje se mejoren. El profesorado, el estudiantado y directivos forman parte de un espacio que es académico y en el cual se suscitan múltiples interacciones con el conocimiento. Cada uno tiene sus propias perspectivas y concepciones, pero finalmente 
deben darse las condiciones de posibilidad para que se logren acuerdos con respecto al logro de las metas afirmativas desde una acción colectiva. Es en este aspecto concreto donde el pensamiento transdisciplinar adquiere una trascendencia e importancia significativa.

Todo esto trata de crear un escenario democrático, en el cual actores universitarios se sienten parte importante de una investigación en la que se les convoca a ser protagonistas. Esto es una forma muy ambiciosa y desafiante de promover pensamiento transdisciplinar desde la cotidianidad y la práctica educativa.

\section{MÉTODo}

La investigación convoca a la totalidad de docentes (6), personas administrativas (10) y estudiantes (16) de los posgrado de la Escuela Superior de Administración Pública (ESAP), (ver Tabla 1) durante los años 2017 y 2018 con reuniones periódicas inicialmente por estamento cada 15 días las primeras tapas y se unen los estamentos para la última etapa que fueron los talleres, la metodología fue transdisciplinaria la cual según Nicolescu (2009), tiene cinco pasos:

TABLA 1

Tipo de participante del estudio

\begin{tabular}{lll|l|l|l}
\hline & & Frecuencia & Porcentaje & $\begin{array}{l}\text { Porcentaje } \\
\text { válido }\end{array}$ & $\begin{array}{l}\text { Porcentaje } \\
\text { acumulado }\end{array}$ \\
\hline Válido & Docente & 6 & 18,8 & 18,8 & 18,8 \\
& Estudiante & 16 & 50,0 & 50,0 & 68,8 \\
& Administrativo & 10 & 31,3 & 31,3 & 100,0 \\
& Total & 32 & 100,0 & 100,0 & \\
\cline { 2 - 6 }
\end{tabular}

Fuente: Elaboración propia

1. El propósito de transformar la realidad social y promover el desarrollo comunitario. Esta problemática genera un viaje de indagación a modo de andadura intelectual (Morin, 1989) que tiene como punto de partida la formulación de un objetivo general, en el que se pretende construir una propuesta pedagógica con la participación de actores universitarios que promuevan un pensamiento transdisciplinar de la enseñanza y el aprendizaje de los posgrados de la ESAP. El logro de este objetivo incluye el planteamiento de tres específicos, en donde el primero remite a determinar la situación problema, con respecto al conocimiento de los actores universitarios de la ESAP sobre el pensamiento transdisciplinar. Este corresponde a una primera etapa de la investigación denominada diagnóstico, en la cual se indaga a través de una encuesta por los pre-saberes que el profesorado, el estudiantado y el personal administrativo han acumulado en su trayectoria profesional, experiencias de aprendizaje o narrativas biográfica a cerca de la transdisciplina y su incidencia en las prácticas de la enseñanza en el saber administrativo de este modo, se identifica la magnitud del problema desde el contexto vital y ecocultural de la escuela de administración; pues una encuesta está constituida por una serie de preguntas dirigidas a una porción representativa de una población, y tiene como finalidad averiguar estados de opinión, actitudes o comportamientos de las personas ante asuntos específicos (Ávila, 2006).

2. La creación de autoconciencia de la realidad social de las personas participantes y de la capacidad de tomar decisiones para mejorarla; se da con el segundo objetivo específico, el cual vincula los saberes y las experiencias de los actores universitarios sobre pensamiento transdisciplinar en la construcción de la propuesta pedagógica, formando parte de una segunda etapa denominada planteamiento de estrategias vinculantes y de acción; se retoman los problemas identificados en el diagnóstico efectuado desde de la institución educativa en donde se evidencia la necesidad de introducir el pensamiento transdisciplinar para comprender la transdisciplina y cimentar la propuesta pedagógica. 
3. Activar a las personas participantes. Capacitar a la gente para solucionar los problemas de su entorno. La técnica de investigación empleada es la sesión en profundidad que crea un espacio de interlocución sistémica, interrecursiva, interretroactiva y psicosocioafectiva para que los actores universitarios se aproximen de manera comprensiva, constructivista y dialógica a la pedagogía y a la transdisciplina como un bucle alternativo para mejorar las prácticas de enseñanza de la administración; Pérez y Alfonzo, (2008) plantean que con estas sesiones se debe permitir la socialización de los saberes en la necesidad de aprender a pensar la realidad.

4. Concientizar, actuar, liberar. Se da con el tercer objetivo y da significado a los aportes concretos del pensamiento transdisciplinar por parte de los actores universitarios en la implementación de la propuesta pedagógica como parte de la malla curricular de los posgrados de la ESAP, encuadra en una última etapa denominada Implementación de la propuesta pedagógica en administración.

5. Desarrollar las capacidades de los miembros, analizar las necesidades, los fines, las demandas, los problemas, las oportunidades. Encontrar soluciones a algunos problemas. Iniciar otras actividades sociales. En este momento de la investigación se elucidan algunas estrategias que enriquecen el desarrollo teórico, metodológico y práctico de la estructura curricular del programa de administración en la universidad, con base en una organización transdisciplinar de los conocimientos, saberes y enfoques en un marco el cruce de intercambio de sinergias comunicacionales entre los actores universitarios. La hipótesis es una propuesta pedagógica de carácter transdisciplinar en los posgrados de la ESAP, Colombia, permitirá mejorar la enseñanza pedagógica de la administración pública en contextos plurales de sociedad y cultura. Este supuesto se confirma con los hallazgos y resultados de la investigación, porque la pedagogización de las prácticas de la enseñanza en una articulación compleja con la transdisciplina ponen en el centro de saber administrativo al ser humano en su multidimensionalidad y restituyen el valor de la pedagogía como el punto central en la gestión colectiva del conocimiento y en la proyección social de la universidad como un organismo eco cultural, vital y que se transforma día a día.

La secuencia de las etapas está enmarcada en los contextos de acción que los actores universitarios agrupados por estamentos definen, propiciando la retroalimentación permanente de los saberes en un proceso deliberativo transdisciplinar y que estimula la deconstrucción y la reconstrucción de los conocimientos aportados por cada participante; para una reforma del pensamiento (Pérez y Alfonzo, 2008).

La encuesta al fundamentarse desde la transdisciplina y los principios sistémicos, hologramático, interrecursivo e interretroactivo de la complejidad no solamente determina el conocimiento y el estado de opinión sobre el pensamiento transdisciplinar, sino que propone interrogantes de alta exigencia intelectual para generar un enunciado escrito de cada actor universitario; en este consignan sus autorreflexiones acerca de las capturas complejas de sus propios pre-saberes y perspectivas. Para la aplicación de la encuesta se elaboró una validación de contenido y de criterio con dos expertos (doctores en pensamiento complejo y pensamiento crítico) y validez de constructo con la aplicación de la encuesta a una muestra representativa según el uso del software SPSS (Cortés y León, 2005).

Las sesiones en profundidad es otra de las técnicas empleadas que produce un clima de aprendizaje colaborativo para abordar los contextos de acción sobre los cuales es posible analizar e interpretar las respuestas argumentales obtenidas durante la aplicación de la encuesta. El desarrollo de estas sesiones posibilita que cada estamento de actores universitarios analice la respuesta argumental en una base de comprensión la cual se concreta en una construcción analítica en la cual se devela el significado de lo que se pretende comunicar al responder cada una de las preguntas de la encuesta.

Los talleres de aprendizaje transdisciplinar son la llave para el cambio y el espacio donde los tres estamentos se integran para construir la propuesta pedagógica con pensamiento transdisciplinar; como afirma Latorre (2008) intenta que el cambio introducido sea asimilado e incorporado a la organización educativa. Lo que supone un cambio en la cultura de la institución que asimila el cambio. 
Desde su estructura y organización el taller es una acción reflexiva de aprendizaje, los talleres educativos son una de las primeras alternativas de enseñanza-aprendizaje frente al método frontal y buscan traer algo de la realidad a la sala de clases.

El corpus de resultados obtenido durante la implementación de las sesiones en profundidad y los talleres de aprendizaje transdisciplinar se le da forma mediante el análisis y la interpretación de la información obtenida. Desde la perspectiva metodológica de esta investigación el análisis se concibe como, un conjunto de instrumentos metodológicos, cada vez más perfectos y en constante mejora, aplicados a discursos (contenidos y continentes) extremadamente diversificados (Bardín, 1991).

El análisis de los datos de la encuesta se enlazo con un adecuado manejo del cuestionario, mediante una construcción de tablas que permita organizar a modo de escalas valorativas los juicios vertidos por los participantes en cada uno de los estamentos (ver Tablas 2,3).

TABLA 2

Definiciones diálogo de saberes

\begin{tabular}{lll|l|l|l}
\hline & & diálogo de saberes & & \\
& & & $\begin{array}{l}\text { intercambio } \\
\text { de }\end{array}$ & posición & \\
& & interdisciplinariedad & conocimiento & \\
dialógica & Total \\
\hline Tipo de & Docente & 2 & 1 & 3 & 6 \\
participante & Estudiante & 3 & 6 & 7 & 16 \\
del estudio & Administrativo & 2 & 5 & 3 & 10 \\
Total & 7 & 12 & 13 & 32 \\
\hline
\end{tabular}

Fuente: Elaboración propia

TABLA 3

Definición de conocimiento

\begin{tabular}{|c|c|c|c|c|c|}
\hline & \multicolumn{3}{|c|}{ Conocimiento } & \multirow[b]{2}{*}{ Total } \\
\hline & & $\begin{array}{l}\text { saber } \\
\text { humano }\end{array}$ & $\begin{array}{l}\text { saber } \\
\text { aprendido }\end{array}$ & $\begin{array}{l}\text { manejo } \\
\text { integral }\end{array}$ & \\
\hline Tipo de & Docente & 1 & 2 & 3 & 6 \\
\hline participante & Estudiante & 6 & 4 & 6 & 16 \\
\hline del estudio & Administrativo & 3 & 4 & 3 & 10 \\
\hline Total & & 10 & 10 & 12 & 32 \\
\hline
\end{tabular}

Fuente: Elaboración propia

Se sintetizan los pasos concretos que se tienen en cuenta para operacionalizar de forma indexada y en escala cualitativa las respuestas al cuestionario (ver Tabla 3 )

El análisis contenido en estas sesiones en profundidad, produce una triangulación ampliada que en secuencia retraen las respuestas argumentales de la encuesta para complementarla con las contribuciones deliberativas en interretroacción durante el diálogo participativo de la sesión; se establece una clara relación entre la reflexión y el planteamiento de estrategias vinculantes de acción para preparar el camino de la construcción de la propuesta pedagógica en la última etapa del proceso investigativo. Es importante señalar que se mantiene como constante procedimental el tratamiento manual de los datos, por tratarse de respuesta escriturales que se someten a un análisis de contenido y a una significación de resultados. 


\section{Discusión}

Las capacidades de pensamiento y de acción desplegadas en este apartado aparece como opciones epistemológicas y pedagógicas para fundamentar el ser, el qué hacer y el saber hacer del profesional de administración.

El profesional de la administración debe ser capaz de integrar dialógicamente en contextos reales de aplicación del cuerpo teórico de conocimientos y de la trama subjetiva que moviliza sus prácticas disciplinares. El administrador deberá entender los axiomas de la transdisciplina que son adaptativos a las nuevas circunstancias de la sociedad y la cultura que los convierten en verdades cambiantes. La palabra axioma deriva del sustantivo griego $\alpha \xi ı \omega \mu \alpha$, la cual significa lo que parece justo o lo que se considera evidente, sin necesidad de demostración. El término viene del verbo griego $\alpha \xi$ เоઘıv (axioein), que significa valorar, que a su vez procede de $\alpha \xi$ ıь (axios), valioso, válido o digno (Carr, 1996).

Los axiomas proporcionan planteamientos comunes que trasversal y dialógicamente trazan hilos de comunicación entre y en el interior de las disciplinas con las que esta establece vincularidades; la identidad, la contradicción y las posibles exclusiones desaparecen para ceder su lugar a lo que Nicolescu, (1996) determinó como, Axioma ontológico, Axioma lógico y Axioma de la complejidad.

El administrador es una persona conectada con el mundo del cual forma parte, no como un conglomerado de asociaciones arbitraria, sino como un complejo anclaje de interacciones complejas en las cuales la estructura de la totalidad de niveles de realidad o percepción, es una estructura compleja, cada nivel es lo que es porque todos los niveles existen al mismo tiempo (Nicolescu, 2006).

El axioma ontológico, del tercero incluido y el de la complejidad, propone un cambio pedagógico en el que el aula se asume como una experiencia de interacción interrecursiva, esto es pertinente para valorar adecuadamente que la pedagogía y la didáctica son actos fundamentalmente dialógicos y comunicativos porque una concepción complexa, abierta y recursiva que facilitase una postura que no se asustase ante la extrañeza del horizonte. Es decir, dejar de naturalizar y por ende de aceptar como inamovible, cada una de las instancias que se dan en las aulas, en las direcciones, en las salas de profesores, en los lugares de recreo (Gonfiantini, 2016).

La persona administradora que se forma en el aula, el personal docente que enseña administración y la persona administrativa que direcciona y planifica la gestión del conocimiento, conforman una especie de tejido complexus, en el que conocer, aprender e interactuar forman una especie de tríada potente para promover la trasformación de la enseñanza y los modos de acceder al conocimiento. Las escuelas de administración son organizaciones interretroactivas e interrecursivas en las cuales las relaciones de aprendizaje no se encuentran mediadas por jerarquías, dispositivos de poder o sociedades disciplinarias.

La administración es un proyecto de institucionalización de un saber, en el cual el principio recursivo lo dimensionan como una instancia significativa en el que el sujeto social produce el proyecto regional alternativo, pero, al mismo tiempo, el proyecto regional alternativo también produce al sujeto (Díaz, Sánchez, Ortiz y Gallardo, 2012).

En esta concepción, el sujeto que aprende y el que enseña están inmersos en un proyecto que se consolida cuando ellos mismos gestionan con recursividad sus saberes y prácticas, por cuanto el pensamiento produce la acción y, de manera simultánea, la acción produce pensamiento, esto significa que la universidad es un todo que se proyecta desde la mismidad individual y a su vez la universidad se interioriza inter-retro-activamente en la concepción subjetiva de la persona, por lo tanto la universidad produce al sujeto-proyecto pero el sujetoproyecto también transforma a la universidad (Díaz et al.2012).

Construir interrecursivamente el conocimiento de la administración es, antes que nada, un proyecto subjetivado de integración multidimensional en el que los actores universitarios son fundamentales para lograr este propósito, la escuela de administración adquiere una fisonomía pedagógicamente humana que la coloca por encima de las instalaciones físicas, los medios audiovisuales, las tecnologías de punta o las distintas 
adecuaciones estructurales que garantizan comodidad y confort. El conocimiento reintegrado produce un sujeto proyecto y un proyecto subjetivado de una universidad a escala humana el cual se presenta como un territorio de acogida y cohabitación entre identidades diversas y singularmente personales.

La pluralidad es una característica fundamental de dichas contextualidad, mientras que la integración inter e intra recursiva es el elemento que potencia los diálogos sociales entre actores y sectores, sujetos y objetos de conocimiento, instituciones educativas y organizaciones sociales, porque la pertinencia técnica y social de los programas de formación alude a la capacidad de la universidad para responder a las necesidades y exigencias de la sociedad (Rojo, 1999).

El administrador público es un sujeto capaz de comprender que la pertinencia de su formación está ligada directamente al liderazgo social que ejerce como profesional y al desarrollo de su perfil administrativo; el saber hacer se configura como un conjunto de comprensiones que no responden tan solo a enfoques macroeconómicos, sino que están vinculados a un objeto formativo el cual se humaniza en la medida que la administración impacta las instituciones y los grupos sociales. Este es un valor agregado de la administración pública en el marco de una educación superior pertinente dado que el concepto de pertinencia comprende así el papel que la educación superior desempeña en la sociedad y lo que esta espera de aquella (Tünnermann, 2015).

La noción de pluralismo multi y sociocultural es un aporte concreto de la complejidad para que el administrador público pueda comprender que su liderazgo y perfil gerencial está inmerso en el tejido social articulador la sociedad es un todo integrado e interdependiente cuya principal característica radica en aceptar que la administración es un lugar de apertura y de encuentro con el otro en donde se acepta la diversidad y se ejecutan acciones de equidad en un entorno transdisciplinar (Rodríguez, 2017).

El administrador público a partir de sus comprensiones va adquiriendo conciencia de la multiplicidad de entornos transdisciplinares y de tejidos socioculturales a los cuales debe enfrentarse como persona profesional y como persona ciudadana al mismo tiempo. Leer críticamente este contexto, desde una perspectiva sistémica es otra capacidad importante que coadyuva en su formación; implica desde lo pedagógico un proceso constructivo en el que estudiantes y docentes aprenden a establecer las relaciones no lineales de una manera reflexiva y recursiva. Esto significa que la lectura del contexto no puede reducirse a la repetición de formulaciones curriculares, por el contrario, ha de ser un conjunto de posibilidades para comprender sistémicamente los saberes, teorías y prácticas de la administración.

El principio sistémico es para la pedagogía un elemento fundamental en el desarrollo de una capacidad para adquirir una concepción sistémica del saber administrativo que requiere un ejercicio analítico de su verdadero significado; y conlleva un acuerdo previo de concebir la realidad en su diversidad y variabilidad, en contraste a una cosmovisión estricta y sencilla (Escobar, 2010). Esta aceptación de la diversidad es lo que nutre la actitud de búsqueda del administrador público en contraste con un aprendizaje que se limita tan solo a la legitimación de los enfoques fraccionados de la tradición administrativa clásica.

La formación transdisciplinaria, es fundamentalmente un ejercicio pedagógico de sistematización de la realidad, a partir del cual la educación superior responde integralmente a las necesidades sociales y a las intencionalidades específicas de los objetos formativos en la administración por cuanto la formación transdisciplinaria en la educación superior es un propósito de gran envergadura (Oliva, Molina, Quintero y Díaz 2018).

Su dimensión conlleva una serie de exigencias. Dos de las más importantes son el enfoque sistémico y la integración de los contenidos, ambas relacionadas entre sí (Pérez, 2003).

Integrar y sistematizar son dos dimensiones que en administración proponen una comprensión amplia de la realidad, sin incurrir en excesos que desborden las fronteras determinadas por la ciencia, la investigación y las prácticas pedagógicas; por esta razón es pertinente precisar que el enfoque sistémico propicia comprender el objeto de estudio como sistema y a su vez como componente de un sistema más amplio. Conduce a captarlo 
en su interacción intrasistémica y con el medio circundante, en su desarrollo, composición, estructura, como un todo no acabado, del cual no se debe absolutizar la conservación de su estabilidad (Pérez, 2003).

Desde la perspectiva de esta investigación, el administrador público desarrolla la capacidad de comprender que los objetos de estudio de su disciplina están incorporados a sistemas mucho más amplios, con los que debe establecer una interacción que es intrasistémica cuando el subjetivamente se posiciona en el saber adquirido y es interactiva si le permite entender que su ejercicio profesional es un todo no acabado.

La capacidad de comprender sistémicamente las relaciones que se articulan entre objetos, sujetos y niveles de interacción entre el administrador, las realidades y los entornos para la administración, es fundamental en el desarrollo de una actitud transdisciplinaria que Carrizo (2005) define en las siguientes palabras:

Se comprende la actitud transdisciplinaria, situada en un multinivel sistémico sobre la relación disciplinar edad/ interdisciplinariedad. Esta actitud permite una mirada que puede -desde el trabajo estrictamente disciplinario, desde el trabajo interdisciplinario y, también, desde el conocimiento extras disciplinarios- comprender las riquezas del diálogo multinivel y horizontal.

La reflexión anterior es clave para dimensionar que el multinivel transdisciplinario es por excelencia articulador e integrador, porque establece la comprensión de la administración como disciplina, lo mismo que el reconocimiento del diálogo como una fuente que ayuda a establecer las interacciones inter y multidimensionales. Esto significa que el administrador no puede quedarse cultivando su propia parcela de conocimiento, ni mucho menos considerarse como el único dueño de la verdad; por el contario debe abrirse a la búsqueda de una actitud transdisciplinaria que fortalezca la capacidad de leer críticamente el mundo como sistema y la realidad como una totalidad fluyente incompleta y no terminada.

Este reposicionamiento del liderazgo y del perfil del administrador encuadra en el sentido de pertinencia de los programas de administración, si se tiene en cuenta que estos pueden quedarse a la saga de las trasformaciones que socialmente se producen en el afuera de las universidades; de ahí que la actitud transdisciplinaria sea relevante para comprender que el enfoque sistémico conduce a generar diálogos cruzados, fertilizaciones y sinergias las cuales hablan de otra perspectiva sistémica-compleja en el tratamiento de asuntos de interés social. Este diálogo cruzado es ni más ni menos que un proceso constructivo y de intercambio sinérgico, el cual desarrolla en el administrador la capacidad de leer críticamente el mundo que lo rodea al mismo tiempo efectuar una lectura crítica de su mismidad y realidad ontológica. Esto es básico para que adquiera herramientas de participaciones los diferentes asuntos de una sociedad y pueda aportar en la socialización de temas tales como la planificación urbana, el tratamiento de las aguas, políticas sanitarias, discriminación, prevención de catástrofes, seguridad, desarrollo económico social, son algunos de los temas en los que una nueva mirada se instala, bajo la exigencia de un tratamiento idóneo y urgente (Carrizo, 2005).

Todas estas temáticas se constituyen en el día a día de la administración y en el marco operativo del administrador público; el como sujeto está instalado en una realidad social que trasversalmente involucra diálogos y debates sobre asuntos que preocupan a los individuos y grupos en determinados contextos culturales y comunitarios; la salud, la educación, la economía, la productividad el comercio, entre otros tópicos son intereses gnoseológicos y prácticos que movilizan el pensamientos administrativo y desarrollan la capacidad de integrar sistémicamente los conocimientos para resolver los problemas que pueden derivarse en los distintos campos, sectores, territorio y contextualidad de la realidad.

El administrador público revalora su papel en la sociedad cuando es capaz de participar activamente en la institucionalización del saber administrativo. Esto significa fijar posturas ideológicas, críticas y epistemológicas con respecto a la razón de ser de la administración y a la pertinencia de sus contenidos; lo más importante es que el administrador en formación o ya formado fortalezca sus capacidades de argumentación como parte del proceso de institucionalización, a este respecto Tapia (2011) afirma que la institucionalización consiste en la adopción de los argumentos administrativos como criterios que estructuran el diseño y funcionamiento de las administraciones públicas y los procesos que materializan una política pública, es así como la Administración de Empresas se ha visto impactada por las megas tendencias 
del cambio, tanto por las variables relacionadas con la era digital y la sociedad del conocimiento, como por el avance en el campo multidisciplinar y la transdisciplina en la construcción y análisis de nuevas políticas (Avendaño y Hernández, 2018).

El pensamiento transdisciplinar es básico para que la argumentación administrativa diseñe e implemente tanto políticas de gestión como modelos, es decir, el argumento es una elaboración intelectual y práctica que sirve de marco para que el conocimiento y los saberes adquieran una fisonomía diferenciada en el contexto donde se genera; la universidad, la empresa el gobierno o el sector privado son entornos administrativos en donde hay que institucionalizar el saber y el conocimiento para que se direccionen los modelos de gestión y se implementen las políticas institucionales. Este marco resignificado garantiza que este proceso sea sistémico, interretroactivo, recursivo y complejamente dinámico.

La transdisciplina en estos principios enunciados, cataliza las dinámicas y provee elementos críticos y alternativos para que estudiantes docentes y administrativos participen sinérgicamente como parte de una microcomunidad científica (Chavarría, 2006); así se revindica no solo el valor del saber administrativo si no la importancia que este tiene para generar trasformaciones sociales.

Las microcomunidades científicas reconocen en los planteamientos herramientas metodológica para construir puentes de convergencia (Walsh, 2005) entre las disciplinas, los enfoques y los modelos de gestión; así puede eludirse la tendencia reduccionista de cercar o parcelar el conocimiento y evitar el riesgo de centralizar los poderes que este genera participar en los proceso de institucionalización del saber es una opción que derrumba las fronteras que imponen las disciplinas y los modelos de gestión administrativa de una política pública, el aislamiento del profesorado en el proceso de construcción de conocimiento educativo y la propuesta de intersección entre la investigación en administración y educación reside en la relación bidireccional que exige el desarrollo de la nueva transdisciplina (Fuentes y Collado 2019).

\section{Resultados}

La pedagogía como parte de la propuesta se concibe como un acto de conciencia en completud fluida, sin la pretensión de binariedad y linealidad de la ciencia clásica. Los axiomas lógicos, ontológico y del tercero incluido de la transdisciplina (Nicolescu, 1996) complejizan el acto pedagógico y aportan una visión distinta de los sujetos que conocen los objetos cognoscibles y los niveles de realidad como una policromía diversificada. Conocer la administración está mucho más allá de una yuxtaposición de teorías enfoques o tendencias e implica una intelección compleja y transdisciplinar del saber administrativo.

El y la administrador/a en formación, el y la administrador/a formado/a y el y la docente administrativo/ a conforman una trama que se teje en complexus, eliminando las distancias entre el enseñante y el aprendiz, conformando microcomunidades académicas científicas y de investigación; estas se encuentran sustentadas en anclajes comunicacionales que cruzan hilos intangibles de creatividad, emoción y afecto sin los cuales no es posible conocer el mundo de la administración y mejorar las prácticas de enseñanza; con base en las respuestas del cuestionario y lo desarrollado durante los talleres y el seminario se formó la propuesta pedagógica se da un ejemplo del análisis elaborado en el área de la humanización de la enseñanza y el aprendizaje, eje fundamental de la propuesta. Con el programa SPSS se organizó el análisis por pregunta lo cual se presenta en la Gráfico 1. 


\section{Gráfico 1}

Humanización en los procesos de formación. Barras simples de problemas prácticos por humanización en procesos de formación académica.

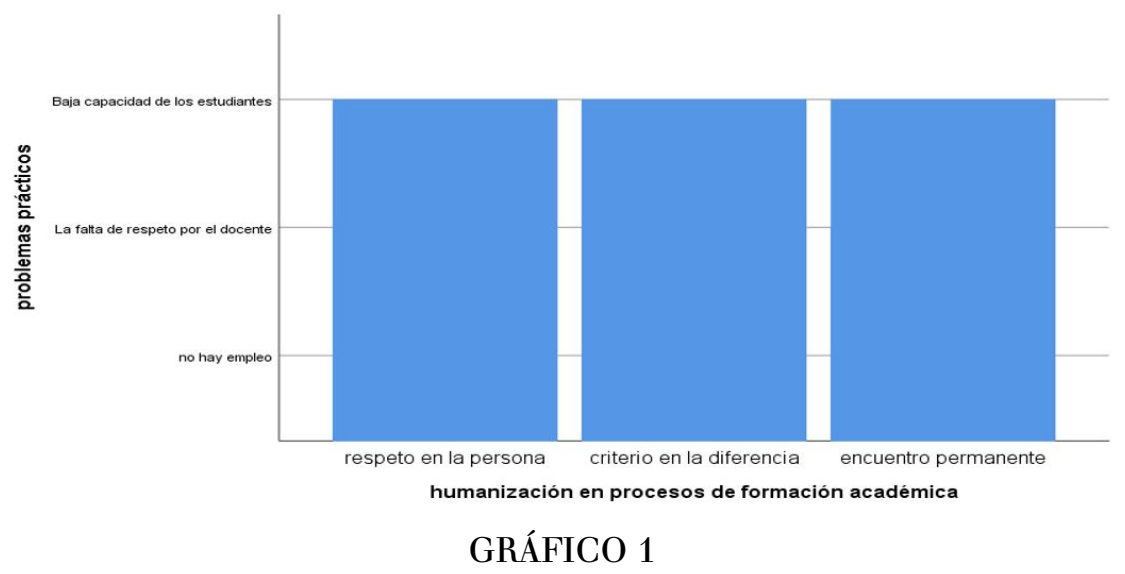

Humanización en los procesos de formación. Barras simples de problemas prácticos por humanización en procesos de formación académica.

Fuente: Elaboración propia

Un ejemplo de cuadro de Cruce de opiniones entre estamentos es el de humanización. 
TABLA 4

Análisis Interestamental. Pregunta: la palabra humanización tiene algún sentido en sus procesos de formación académica en su área disciplinar

\begin{tabular}{|c|c|c|}
\hline ADMINISTRATIVOS & DOCENTES & ESTUDIANTES \\
\hline $\begin{array}{l}\text { La humanización } \\
\text { en la formación } \\
\text { administrativa es } \\
\text { importante porque } \\
\text { ayuda a entender la } \\
\text { forma como las } \\
\text { demás personas } \\
\text { piensan y miran el } \\
\text { mundo desde su } \\
\text { propia perspectiva } \\
\text { y asi se pueden } \\
\text { brindar } \\
\text { herramientas para } \\
\text { formar mejores } \\
\text { personas para la } \\
\text { sociedad. Los } \\
\text { enunciados ponen } \\
\text { de relieve que la } \\
\text { formación debe } \\
\text { resolver las } \\
\text { problemáticas } \\
\text { sociales sin } \\
\text { constreñimientos } \\
\text { Culturales; el } \\
\text { humanismo en la } \\
\text { formación de los } \\
\text { nuevos académicos } \\
\text { recupera valores } \\
\text { como la honestidad } \\
\text { que son } \\
\text { fundamentales en } \\
\text { concebir la } \\
\text { formación desde la } \\
\text { calidad humana y } \\
\text { el servicio a la } \\
\text { ciudadania. Se } \\
\text { reivindica en } \\
\text { algunos enunciados } \\
\text { la importancia de la } \\
\text { formación como } \\
\text { responsabilidad } \\
\text { con el medio } \\
\text { ambiente y con el } \\
\text { mundo de la vida } \\
\text { de las } \\
\text { comunidades. }\end{array}$ & $\begin{array}{l}\text { El } \\
\text { profesorado } \\
\text { tiene claridad } \\
\text { en el manejo } \\
\text { del concepto } \\
\text { de } \\
\text { humanización, } \\
\text { lo consideran } \\
\text { básico para } \\
\text { trabajar } \\
\text { junto, } \\
\text { poniendo en } \\
\text { el centro de la } \\
\text { administración } \\
\text { al hombre en } \\
\text { su dignidad } \\
\text { personal, para } \\
\text { generar } \\
\text { educación y } \\
\text { desarrollo. } \\
\text { Humanizar es } \\
\text { para ellos } \\
\text { importante en } \\
\text { fortalecer } \\
\text { capacidades } \\
\text { que desde la } \\
\text { administración } \\
\text { acentúa la } \\
\text { importancia } \\
\text { de una } \\
\text { educación que } \\
\text { forme } \\
\text { acciones } \\
\text { integrales de } \\
\text { la realidad y } \\
\text { la historia del } \\
\text { país. La } \\
\text { humanización } \\
\text { implica } \\
\text { comprender } \\
\text { que el sujeto } \\
\text { que se forma } \\
\text { en la escuela } \\
\text { de la } \\
\text { administración } \\
\text { es } \\
\text { conocimiento } \\
\text { y discurso } \\
\text { que se vincula } \\
\text { con el otro en } \\
\text { un } \\
\text { permanente } \\
\text { juego de } \\
\text { comunicación, } \\
\text { a través con el } \\
\text { encuentro } \\
\text { permanente } \\
\text { con estos } \\
\text { sujetos en el } \\
\text { marco de la } \\
\text { habitantica } \\
\text { social. } \\
\text { and }\end{array}$ & $\begin{array}{l}\text { Los } \\
\text { enunciados } \\
\text { que se analiza } \\
\text { dejan claro } \\
\text { que en la } \\
\text { actualidad es } \\
\text { necesario } \\
\text { humanizar el } \\
\text { saber en la } \\
\text { administración } \\
\text { pública. Esto } \\
\text { implica un } \\
\text { respeto por el } \\
\text { hombre y la } \\
\text { sociedad } \\
\text { como parte de } \\
\text { un proceso } \\
\text { que potencia } \\
\text { el desarrollo } \\
\text { de estos dos } \\
\text { conceptos. La } \\
\text { ética, los } \\
\text { valores y las } \\
\text { actividades de } \\
\text { servicio a la } \\
\text { comunidad } \\
\text { son para el } \\
\text { estudiantado } \\
\text { elementos } \\
\text { necesarios en } \\
\text { la formación } \\
\text { humana de } \\
\text { los nuevos } \\
\text { profesionales } \\
\text { en } \\
\text { administración }\end{array}$ \\
\hline
\end{tabular}




\section{Fuente: Elaboración propia}

En la propuesta pedagógica que efectuaron los actores universitarios, la universidad es un espacio ecocultural y vital el cual se forja desde la mismidad, ellos actúan como sujetos que se preparan a sí mismos en un proyecto pedagógico común, que se elabora sobre la base de la solidaridad intersubjetiva. Las ideas y los conceptos aportados por los actores convierten a la universidad en un territorio de acogida que responde a las necesidades sociales y que se retroalimentan en doble vía de los contextos de realidad. La pluralidad transdisciplinaria le permite a la institución migrar hacia mutaciones significativas para responder a los desafíos que se plantean desde un mundo cambiante (ver Tabla 4).

La propuesta académica construida a partir de la investigación acción desarrollada se fundamentó en las epistemologías del sur y la educación popular como dos corrientes orientadoras del trabajo. Posteriormente se finaliza con el aporte del pensamiento complejo y la transdisciplina como herramientas (Vanoli, 2017).

La propuesta se estructura desde cuatro componentes, de los cuales el primero hace explícita la relación que se da entre pensamiento transdisciplinar, complejidad y la pedagogía como un territorio en construcción de prácticas humanizadora. Esta es una idea que organiza una concepción sistémica transdisciplinar en la cual el actor de la administración es fundamental para que la ciencia recupere su razón de ser como generadora de cambios y transformaciones en el mundo social; solo así es posible lograr una enseñanza que restituya la axiología, la ética y la responsabilidad que orienta el quehacer administrativo en los diferentes contextos de realidad donde este se institucionalice.

El segundo componente establece que la propuesta fortalece en los actores educativos capacidades de pensamiento y acción transdisciplinar, que sin lugar a dudas potencian un conjunto de habilidades en Interretroacción sistémica para que la enseñanza se resignifique desde una pedagogía de la emancipación y de la construcción afectiva en el aula. Estas capacidades proyectan un docente, un administrativo y un estudiante que sea capaz de Integrar dialógicamente los axiomas de la transdisciplina, construir una visión interrecursiva del saber administrativo, comprender la pluralidad y la multiculturalidad como el caudal sociocultural de la administración pública, leer crítica y sistémicamente el contexto de realidad y revalorar el papel del administrador público desde su participación en los procesos de institucionalización del saber administrativo.

El tercer componente desarrolla la apertura metodológica, esta es una andadura intelectual e investigativa que en la enseñanza de la administración se hace necesaria para fortalecer las microcomunidades de aprendizaje y de investigación. Abrirse metodológicamente articula la noción del método como construcción democrática, y admite que la heterogeneidad no simplificada es un elemento necesario para generar conocimiento nuevo en administración. La metodología se asume como un campo fecundo de interacciones objetuales y subjetivas que descubre nuevos caminos para la producción del conocimiento. Esta percepción acoge opciones multimetódica, enfoques cualitativos y cuantitativos y una variedad de tendencias y posturas frente a lo que es producir, apropiar y distribuir socialmente el conocimiento en administración.

El cuarto componente une la perspectiva pedagógica y la humanización como aspectos sustanciales en la fundamentación transdisciplinar de la propuesta. Esta relación se potencia en el bucle pedagogía y humanización, en la concepción rizomática del acto pedagógico en administración, en la triada racionalidad emancipación y excelencia, en el binomio ética y responsabilidad social y en la transferencia de sinergias comunicativas en la humanización del saber administrativo.

Estos enlaces biopsicoecoculturales convierten la enseñanza en un campo fertilizado de interacciones humanas que crean puentes de convergencia para que los actores universitarios se miren a los ojos, se abracen solidariamente y compartan las transacciones afectivas que estimulan la calidez como una virtud necesaria para pedagogizar el acto educativo y renovar la esperanza de los desposeídos afectivamente.

Estas lecturas del contexto de realidad abren el camino para que el administrador público pueda leer críticamente el entorno institucional, a este respecto, la Tabla 2 muestra la inferencia que analizó este tópico de indagación. 
La inferencia comprende que el contexto institucional no es el más favorable porque se encuentra afectado por el declive ético y moral de algunas actuaciones de los administradores públicos; esto puede generar en algún momento desconfianza en las instituciones administrativas y en su ejercicio profesional. La transdisciplinariedad es vista como una herramienta metodológica para que la apropiación institucional del saber se confronte en distintos actores, lugares, teorías y prácticas, siendo esto primordial para nutrir el diálogo de saberes de la administración y mejorar la percepción que se tiene de esta. El saber administrativo se apropia socialmente y genera nuevos lenguajes que si son filtrados por una perspectiva ética y moral contribuye significativamente en la restauración axiológica y práctica del papel del administrador público.

\section{CONCLUSIONES}

La investigación propone un marco cognitivo para una comprensión transdisciplinaria de la administración pública, en su concepción vital con la pedagogía como un terreno de la humanización de la enseñanza y de las prácticas pedagógicas. Los actores universitarios aportan sus perspectivas con respecto a la articulación de los saberes, las técnicas, normas método y contenidos que se encuentran instaurados en el fondo de una disciplina que a modo de diafragma se abren para capturar significativamente los niveles lógicos, ontológicos y del tercero incluido de una realidad que es sustancialmente policromática. El terreno sobre el cual actúan los administradores en formación y los ya formados, los administrativos y las escuelas de administración es de una fisonomía diversificada y que presenta un relieve irregular en su geografía política, social, cultural y económica.

La práctica educativa donde se instauran la enseñanza, la pedagogía y la investigación como parte de la propuesta pedagógica con pensamiento transdisciplinar, conduce a establecer que la escuela de administración debe ser un proyecto subjetivado individualmente y que socialmente subjetiva un proyecto pedagógico común.

El conocimiento transdisciplinar contribuye en que la escuela de administración se asuma como un escenario de acogida, en donde los actores universitarios comprenden, interpretan e interactúan con una realidad que es compleja y cambiante. Acoger implica que la enseñanza sea revalorada por la pedagogía como la médula de los procesos para gestionar transdisciplinariamente los saberes y tejer cooperativamente los vínculos afectivos, emocionales y comunicativos que son básicos en la configuración de un territorio que debe habitarse en convivencia. Lo más importante es capturar los nuevos lenguajes emergentes de la transdisciplina, involucrando a los actores de la administración en nuevas dinámicas de construcción de conocimiento y mediación pedagógica, para que la enseñanza y el aprendizaje se retroalimenten como sistemas interretroactivas y recursivos que se potencian desde una profunda reflexión compleja y transdisciplinaria.

Hay un logro de sinergia comunicativa entre la persona docente investigadora y los actores universitarios; este es el que propicia una dinámica grupal que fortalezca las capacidades de autoestudio y autoconstrucción del saber administrativo en un proceso de exigencia cognitiva asumido como un viaje de indagación por la transdisciplina como un territorio que se recorre para mejorar el modo como se construye socialmente el conocimiento y la manera como este se institucionaliza en la escuela de administración para mejorar las prácticas de enseñanza.

Los actores universitarios aportan sus perspectivas con respecto a la articulación de los saberes, las técnicas, normas método y contenidos que se encuentran instauradas en el fondo de una disciplina que a modo de diafragma se abren para capturar significativamente los niveles lógicos ontológicos y del tercero incluido de una realidad que es sustancialmente policromática. 
Ricardo Antonio Escobar, et al. Transdisciplina, Enseñanza en Posgrados, Escuela Superior De Admin...

\section{ReFERENCIAS}

Álvarez C., (2010). Representando la ESAP: ¿qué ha sido, qué es hoy y qué debería ser? Bogotá: Escuela Superior de Administración Pública.

Ávila, H. (2006). Introducción a la metodologia de la investigación. México DF, México: eumed net.

Avendaño, N., y Hernández, L. Á. (2018). Líneas y tendencias de la administración de empresas como disciplina desde lo Internacional, Nacional y Local (Tesis de doctorado). Corporación Universitaria Minuto de Dios, Cundinamarca, Colombia.

Bardín, L. (1991). Análisis de contenido. Madrid, España: Ediciones AKAL.

Benítez, J. L. S., y López, S. G. (2017). La complejidad y la transdisciplina como Sistemas teóricos en el hacer de la educación superior. Educación y Humanismo, 19(33), 305-319.

Carr, W. (1996). Una teoría para la educación: hacia una investigación educativa Crítica, Madrid, España: Ediciones Morata.

Carrizo, L. (2005). Gestión social del conocimiento [Mensaje en un blog]. Future learn. Recuperado de https://bit. ly/2MVSZjp

Chavarría, J. (2006). Teoría de las situaciones didácticas. Rev. Cuadernos de Investigación y Formación en Educación Matemática, 1(2), 1-10.

Chaves, J.M.P. (2010). Consideraciones básicas del pensamiento complejo de Edgar Morin, en la educación. Revista Electrónica Educare, 14(1), 67-75.

Codina, P., Sotolongo, L. y Díaz, C. (2006). La revolución Contemporánea del saber y la complejidad social: hacia unas ciencias sociales de nuevo tipo. Texas, México: CLACSO.

Cortés, E. y León, M. (2005). Generalidades sobre metodología de la investigación. Ciudad del Carmen, México: Universidad Autónoma del Carmen.

Díaz, G., Sánchez, M., Ortiz, C., Gallardo, R. (2012) ¿Torbellinos? Los intersticios en la construcción del Desarrollo regional alternativo. Interdisciplina y transdisciplina: aportes desde la investigación y la intervención social universitaria. Centro Interdisciplinario para la Formación y Vinculación Social, 1(1), 47-71

Escobar, Y. C. (2010). Interdisciplinariedad: Desafío para la Educación Superior y La Investigación. Revista Luna Azul, 1(31), 156-169.

Escobar, R. A., y Escobar, M. B. E. (2016). El pensamiento transdisciplinar en la enseñanza de la administración una pesquisa por su estado del arte entre el año 2000 y 2015. Sinapsis, 8(1), 1-20.

Espinosa, A. C. (2013). Estrategias metodológicas para operacionalizar la Práctica educativa transdisciplinaria, en conjunto Con los actores universitarios, en las licenciaturas del Centro de Estudios Universitarios Arkos de Puerto Vallarta, Jalisco, México (Tesis Doctoral). Universidad Estatal a Distancia, San José, Costa Rica.

Fuentes, A., y Collado, J. (2019). Transdisciplinary epistemological foundations of education and neuroscience. Sophia Colección de Filosofía de la Educación (26), 83-113.

García, R. (2006). Sistemas complejos: conceptos, método y fundamentación Epistemológica de la investigación interdisciplinaria. Barcelona, España: Gedisa.

Gonfiantini, V. (2016). Formación docente y diálogo de saberes en el Kairos Educativo. Sophia. Colección de Filosofía de la Educación, 1(21), 229-245.

Latorre, A. B. (2008). La investigación-acción: Conocer y cambiar la práctica Educativa. Málaga, España: Grao.

Mayo, E. (1946). Problemas humanos de una civilización industrial. Buenos Aires, Argentina: Galatea-Nueva Visión.

McGregor, D. (1969). El aspecto humano de las empresas. México DF, México: Diana.

Morin, E. (1989). Los cambios de la vida intelectual francesa. Revista Foro, 8(1), 61-72.

Morin, E. (1998). Introducción al pensamiento complejo. Madrid, España: Gedisa.

Morin, E. (2004). Los siete saberes necesarios para la educación del futuro. Madrid, España: Grupo Planeta (GBS). 
Musgrave, G. L., y Elster, R. S. (1974). Management by objetives and goal Setting. Recuperado de http://archive.org/ details/managementbyobje577musg

Nicolescu, B. (1996). La Transdisciplinariedad. [Mensaje en un blog]. Mónaco: Du Rocher. Recuperado de https://b it.ly/2q1BC81

Nicolescu, B. (2006). Transdisciplinariedad: pasado, presente y futuro. Visión docente Con-ciencia, 5(31), 1-15.

Nicolescu, B. (2009). La transdisciplinariedad: manifiesto. Edgar Morin el padre del pensamiento complejo website Recuperado de https://bit.ly/34SfqvW

Odiorne, G.S. (1995). Administración por objetivos: nuevo sistema para la Dirección. México DF, México: Limusa.

Oliva, I. G., Molina, W. M., Quintero, J. J., y Díaz, N. S. (2018). Prospectiva transdisciplinaria y organización del conocimiento: Una aproximación epistémica a la educación superior en la Región de Magallanes, Chile. Magallania (Punta Arenas), 46(2), 129-142.

Pérez, C. R. (2003). Paradigma de la complejidad, modelos científicos y Conocimiento educativo. Ágora digital, 1(6), $1-10$.

Pérez, E. y Alfonzo N. (2008). Diálogo de saberes y proyectos de Investigación en la escuela. Educare, 12(42), 456-460. Recuperado de http://www.redalyc.org/resumen.oa?id=35614569005

Ponce, A., (1985). Administración por objetivos. México DF, México: Limusa.

Pozzoli, M. T. (2006). El sujeto de la complejidad. La construcción de un Modelo Teórico Transdisciplinar (eco-psicosocio-histórico-educativo). Polis. Revista Latinoamericana, 1(15), 2-14 Recuperado de http://journals.opened ition.org/polis/4921

Rodríguez, A. (2017). La transdisciplina y el pluralismo sociológico en el Conocimiento, la ciencia y la tecnología. Revista Digital Internacional de Psicología y Ciencia Social, 2(2), 159-169. doi: https://doi.org/10.22402/j.rdip ycs.unam.2.2.2016.136.159-169

Rojo, N. (1999). Pertinencia social de los programas académicos de perfil Gerencial en el sector salud. Educación Médica Superior, 13(1), 70-79.

Steiner, G., (1985). Planeación estratégica: lo que todo director debe saber. México DF, México: Compañía Editorial Continental.

Tapia E. (2011). La configuración del saber administrativo Público. Un análisis desde el rol de las doctrinas en la argumentación Administrativa. Administración y Desarrollo, 39(53), 109-122.

Taylor, F.W., Fayol, H., y Lender, H. H. (2003). Principios de la administración Cientifica. Madrid: Edigrama.

Tünnermann, C. B. (2015). Pertinencia social y principios básicos para orientar el diseño de políticas de educación superior. Temas Nicaragüenses, 89(75), 75-91.

Vanoli, F. N. (2017). El pensamiento complejo y la transdisciplina en el Abordaje del hábitat: Reflexiones en torno a la experiencia del Seminario de Gestión Territorial y Hábitat.Revista Pensum, 3(3), 141-146.

Vélez, S. (2011). Una mirada a los nuevos enfoques de la gestión Pública. Administración y Desarrollo, 39(53), 57-74.

Walsh, C., (2005). Pensamiento crítico y matriz (de) colonial. Quito, Ecuador: Abya Yala.

Weber, M., (1994). Economía y sociedad: Tipos de dominación. México DF, México: Fondo de Cultura económica.

\section{BY-NC-ND}

